\title{
Microclima de vinhedos sob cultivo protegido
}

\author{
Microclimate of vineyards under protected cultivation
}

\section{Geraldo ChavarriaI Loana Silveira CardosoII Homero BergamaschiI Henrique Pessoa dos Santos ${ }^{\text {III }}$

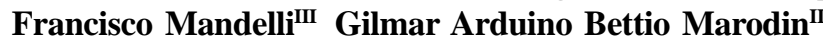

RESUMO

Alterações microclimáticas em vinhedos, provocadas pelo uso de cobertura plástica, interferem na fisiologia das plantas e na incidência de doenças fúngicas em videiras. Assim, o objetivo deste trabalho foi avaliar a influência da cobertura plástica no microclima de vinhedos, em particular na qualidade da radiação solar. $O$ experimento foi conduzido nos ciclos 2005/06 e 2006/07, em Flores da Cunha, Rio Grande do Sul (RS), em um vinhedo de 'Moscato Giallo' conduzido

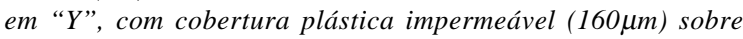
12 fileiras com $35 \mathrm{~m}$, deixando-se cinco fileiras sem cobertura (controle). Em ambas as áreas, avaliou-se o microclima quanto à temperatura do ar, umidade relativa do ar, radiação fotossinteticamente ativa e velocidade do vento, próximo ao dossel vegetativo e aos cachos. Medições contínuas foram efetuadas utilizando sensores e sistemas automáticos de aquisição de dados. Alterações na qualidade da radiação solar incidente sobre o dossel vegetativo, no espectro de 300 a $750 \mathrm{~nm}$, foram avaliadas por meio de medições durante cinco dias, com espectroradiômetro. A cobertura plástica impermeável à água sobre as fileiras das plantas aumentou a temperatura do ar e diminuiu a radiação fotossinteticamente ativa e a velocidade do vento. A cobertura interferiu na qualidade da radiação solar incidente, principalmente, reduzindo a irradiância na faixa do ultravioleta e a razão entre a radiação nas faixas do vermelho e vermelho-distante.

Palavras-chave: Vitis vinifera, plasticultura, micrometeorologia, cultivo protegido.

\section{ABSTRACT}

Microclimate alterations promoted by plastic covering over vineyards interfere in the plant physiology and fungal diseases incidence on grapevines. The aim of this research was to evaluate the influence of the plastic covering on the microclimate of vineyards, in particular on the quality of the incoming solar radiation. The experiment was carried out in 2005/06 and 2006/07 seasons in Flores da Cunha-RS, in a vineyard of Moscato Giallo cultivar shaped in $Y$, with impermeable plastic $(160 \mu \mathrm{m})$ over 12 rows of $35 \mathrm{~m}$ length and five rows without covering (control). In both treatments the air temperature and humidity, incoming photosynthetically radiation and wind speed were measured at the level of the canopy and clusters. Continuing measurements were taken through sensors and automatic acquisition systems (datalogger). Influences of the covering on quality of the incoming solar radiation, from 300 to $750 \mathrm{~nm}$, were evaluated through a spectroradiometer. The impermeable plastic covering above the plant rows increased the air temperature and decreased the photosynthetically radiation and wind speed. The covering interfered on the quality of the incoming solar radiation, by reducing mainly the irradiance in the ultraviolet band and reducing also the ratio between the irradiance in the red and far-red bands.

Key words: Vitis vinifera, plasticulture, micrometeorology, protected cultivation.

\section{INTRODUÇÃO}

Em regiões com elevada probabilidade de ocorrência de chuvas no período de maturação das uvas, como a Serra Gaúcha, é observada com frequência a realização de colheitas antecipadas, em comparação ao ponto ideal de maturação. Essa prática tem sido realizada com o intuito de evitar perdas ocasionadas por podridões dos frutos, porém resulta no comprometimento da qualidade enológica do mosto

IUniversidade de Passo Fundo (UPF), CP 611, 99001-970, Passo Fundo, RS, Brasil. E-mail: geraldochavarria@upf.br. Autor para correspodência.

"Universidade Federal do Rio Grande do Sul (UFRGS), Porto Alegre, RS, Brasil.

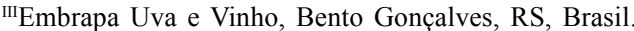


pela paralisação do processo de maturação (TONIETTO \& FALCADE, 2003). O cultivo protegido pela modificação microclimática que pode produzir se torna uma ferramenta, nessas condições de excesso de chuva, capaz de diminuir a incidência de doenças fúngicas e o número de aplicações de fungicidas (CHAVARRIA et al., 2007).

A utilização de coberturas plásticas no cultivo de plantas pode exercer grande influência sobre as condições microclimáticas. De acordo com CARBONNEAU (1984), em vinhedos existem dois tipos de microclima: 1) natural - correspondente às superfícies da ordem de 10 a $100 \mathrm{~m}^{2}$; 2) microclima da planta caracterizado por variáveis climáticas no entorno de cada planta. Porém, as variáveis micrometeorológicas estão estreitamente ligadas à quantidade e distribuição de folhas no espaço (TEIXEIRA \& LIMA FILHO, 1997).

Estudos recentes demonstram que a cobertura plástica altera alguns parâmetros de microclima do vinhedo, em particular as temperaturas máximas, a disponibilidade de radiação solar e a presença de água livre sobre as folhas (FERREIRA et al., 2004; CARDOSO et al., 2008). Uma das principais interferências causadas pela cobertura plástica é o aumento da temperatura relacionado à redução da velocidade do vento, que diminui a perda de calor devido à menor movimentação de ar (SEGOVIA et al., 1997). No caso de estufas plásticas, a temperatura do ar interior difere da externa, dependendo da densidade de fluxo de radiação solar incidente no interior destas e do seu manejo (FERREIRA et al., 2004). Segundo SEEMAN (1979), a alteração da temperatura do ar também depende do tamanho da estufa e do volume de ar a ser aquecido.

Em cultivo protegido com abertura lateral para videira 'Cabernet Sauvignon', em Jundiaí, São Paulo (SP), foi observado que as temperaturas máximas e mínimas do ar, nesse ambiente parcialmente modificado, foram mais elevadas que no cultivo convencional a céu aberto (FERREIRA et al., 2004). Na região conhecida como Serra Gaúcha, CARDOSO et al. (2008) destacaram aumento das temperaturas pelo uso de cobertura plástica, com maior efeito no período diurno e menor sobre as temperaturas noturnas.

No uso da cobertura plástica no cultivo de videiras, a velocidade do vento é substancialmente reduzida (CARDOSO et al., 2008). Sabe-se que a ação dos ventos é importante no cultivo da videira, assim como em outras culturas, por alterar a condição térmica do vinhedo, podendo causar danos mecânicos e inibição fisiológica foliar pelo fechamento estomático (PEDRO JÚNIOR et al., 1998).

A radiação solar que atinge a planta é reduzida pela cobertura plástica. Em algumas regiões do Brasil, tem crescido o uso de coberturas na agricultura, buscando a atenuação da radiação solar e possibilitando o cultivo, principalmente de olerícolas em épocas com alta disponibilidade energética. A atenuação da radiação solar pelas coberturas é importante, pois afeta outros componentes do balanço energético, como os fluxos de calor sensível e latente, além da condição hídrica das plantas e do processo fotossintético (PEZZOPANE et al., 2004).

Estudando o efeito da cobertura plástica de polietileno de baixa densidade $(160 \mu \mathrm{m})$, CARDOSO et al. (2008) observaram interceptação média de $30 \%$ na radiação fotossinteticamente ativa incidente sobre o dossel vegetativo. Contudo, sabe-se que essa atenuação da radiação é variável de acordo com o ângulo de incidência dos raios solares na cobertura (SENTELHAS et al., 1997), com a transmitância (CRITTEN \& BALLEY, 2002), com o tempo de uso da cobertura (REIS \& CARRIJO, 1999; VENTURIN \& SANTOS, 2004) e com a cor do filme plástico utilizado (SENTELHAS et al., 1997).

Detalhamentos das modificações que o cultivo protegido pode exercer sobre o microclima da videira são de grande relevância, considerando que todas essas mudanças influenciam o rendimento e a qualidade das uvas. Dessa forma, os objetivos do presente trabalho foram avaliar a influência da cobertura plástica sobre o microclima de vinhedos de Moscato Giallo e caracterizar seus efeitos sobre a qualidade da radiação solar.

\section{MATERIAL E MÉTODOS}

O experimento foi conduzido durante os ciclos de 2005/06 e 2006/07 (desde a poda de inverno até 30 dias após a colheita das uvas, 1aㅗ safra - 02/03/06 e 2a safra - 22/02/07), em um vinhedo comercial localizado em Flores da Cunha-RS, situado à latitude $29^{\circ} 06^{\prime} \mathrm{S}$, longitude $51^{\circ} 20^{\prime} \mathrm{W}$ e altitude de $541 \mathrm{~m}$. Utilizaram-se plantas da cultivar 'Moscato Giallo' (Vitis

Ciência Rural, v.39, n.7, out, 2009. 
vinifera $\mathrm{L}$.) com sete anos de idade (clone VCR1), com porta-enxerto 'Kober 5BB', com espaçamentos de 3,0m entre plantas e $0,9 \mathrm{~m}$ na linha. As linhas estavam na direção sudeste-noroeste, conduzidas em "Y" e deixando-se, em média, quatro ramos (varas) de quatro a seis gemas e oito esporões de duas gemas por planta. O solo é um Neossolo litólico, segundo a classificação de STRECK et al. (1999). O clima da região é classificado como temperado, do tipo fundamental $\mathrm{Cfb}$, de acordo com a classificação climática de KÖPPEN (1936).

$\mathrm{O}$ vinhedo foi dividido aleatoriamente em duas partes, sendo uma das partes com 12 fileiras cobertas na linha de cultivo com lonas de polipropileno trançadas, transparentes e impermeabilizadas com polietileno de baixa densidade, com $160 \mu \mathrm{m}$ de espessura e largura de $2,65 \mathrm{~m}$. Na outra parte, foram mantidas cinco fileiras descobertas, cujas linhas centrais foram consideradas como plantas-controle. As plantas apresentavam estatura média de $1,5 \mathrm{~m}$ e as coberturas ficaram a uma altura de $\pm 100 \mathrm{~cm}$, acima da posição central do dossel vegetativo, e $\pm 20 \mathrm{~cm}$ em cada extremidade, constituindo um $\mathrm{V}$ invertido sobre um dossel em Y

Nas áreas com e sem cobertura plástica, o microclima foi avaliado na altura do dossel vegetativo e no nível dos cachos. Essa avaliação foi realizada através de medições de temperatura e umidade relativa do ar com psicrômetros de pares termoelétricos, velocidade do vento com anemômetros de conchas $(50 \mathrm{~cm}$ acima do dossel vegetativo) e radiação fotossinteticamente ativa $(400-700 \mathrm{~nm})$, com barras de $1,20 \mathrm{~m}$ equipadas com cinco células fotovoltáicas de silício amorfo. Todos sensores foram conectados a sistemas de aquisição de dados (dataloggers CR10 e CR21X, Campbell ${ }^{\circledR}$ ). Ambos os sistemas foram programados para efetuar leituras a cada minuto e médias a cada 30 minutos. A influência da cobertura na radiação solar incidente ao nível do dossel, na faixa de 300 a 750nm, foi avaliada por meio de cinco medições com espectroradiômetro da marca Li-Cor Inc., modelo LI-1800, nos dias 11/01/06, 13/02/06, 06/03/06, 19/01/07 e 28/03/07 e pela média aritmética.

Utilizou-se o programa R (R-Project, 2007) para análise estatística dos elementos micrometeorológicos (temperatura média, mínima e máxima do ar; umidade relativa do ar; radiação fotossinteticamente ativa e velocidade do vento), e as diferenças entre os tratamentos foram analisadas por regressão linear em função da área descoberta. A significância do coeficiente angular das equações resultantes foi avaliada pelo teste t. $\mathrm{Na}$ análise dos dados de radiação fotossinteticamente ativa e velocidade do vento, o intercepto foi fixado em zero, pois sempre que a medida externa for nula a interna deve também ser nula.

$\mathrm{Na}$ análise da interferência da cobertura sobre o espectro de radiação solar, os dados foram submetidos à análise de variância, e as médias foram comparadas pelo teste de Tukey em nível de 5\% significância.

\section{RESULTADOS E DISCUSSÃO}

Nas avaliações das condições micrometeorológicas, nos dois ciclos (2005/06 e 2006/ 07), foram observadas alterações ocasionadas pelo o uso da cobertura plástica sobre as fileiras de cultivo (Tabela 1). Verificou-se que, à medida que a temperatura máxima diminuiu fora da cobertura, a redução correspondente sob a cobertura foi menor. Isso pode ser verificado pelo coeficiente angular de regressão, altamente significativo $\left(0,897 ; \mathrm{P}_{\beta=1}=0,0005\right)$, o qual indica que, para cada grau Celsius de redução na temperatura externa, a temperatura abaixo da cobertura cai $0,9^{\circ} \mathrm{C}$. Em função do aumento da temperatura máxima, a temperatura média teve padrão similar $(0,96$; $\left.\mathrm{P}_{\beta=1}=0,0265\right)$. Contudo, foi observado que as temperaturas mínimas diárias tiveram menor incremento, verificado pelo coeficiente de regressão $\left(0,972 ; \mathrm{P}_{\beta=1}=0,0012\right)$ (Tabela 1). O aumento das temperaturas máximas foi observado também por FERREIRA et al. (2004), analisando vinhedo de 'Cabernet Sauvignon' com cobertura plástica nas linhas de cultivo em Jundiaí, São Paulo (SP).

De forma geral, ficou evidenciado que, sob cobertura plástica, a perda de calor é retardada em relação ao vinhedo a céu aberto, resultando em maior amplitude térmica. SEGOVIA et al. (1997) consideraram a menor movimentação de ar como o principal fator dessa maior amplitude térmica no ambiente protegido. Isso pode ser sustentado nos resultados obtidos, pois a velocidade do vento foi atenuada em $90 \%$ (coeficiente angular de 0,107; $\left.\mathrm{P}_{\mathrm{B}=1}<0,0001\right)$ junto ao dossel vegetativo das plantas cultivadas sob cobertura plástica (Tabela 1). 
Tabela 1 - Temperatura e umidade relativa do ar, radiação fotossinteticamente ativa (RFA) e velocidade do vento em vinhedo da cultivar 'Moscato Giallo' com (C) e sem (D) cobertura plástica, obtidos durante os ciclos 2005/06 e 2006/07. Flores da Cunha, RS.

\begin{tabular}{|c|c|c|c|c|c|}
\hline \multirow{2}{*}{ Variáveis microclimáticas } & \multicolumn{2}{|c|}{-------2005/2006-------- } & \multicolumn{2}{|c|}{------2006/2007------- } & \multirow{2}{*}{ Equações de regressão** } \\
\hline & $\mathrm{C}$ & $\mathrm{D}$ & $\mathrm{C}$ & $\mathrm{D}$ & \\
\hline Temperatura máxima no dossel $\left({ }^{\circ} \mathrm{C}\right)$ & 31,94 & 28,19 & 31,18 & 30,37 & $\mathrm{C}=4,51+0,897 \mathrm{D} \mathrm{R}^{2}=0,74$ \\
\hline Temperatura média no dossel $\left({ }^{\circ} \mathrm{C}\right)$ & 21,28 & 20,28 & 21,91 & 21,84 & $\mathrm{C}=1,37+0,960 \mathrm{D} \mathrm{R}^{2}=0,95$ \\
\hline Temperatura mínima no dossel $\left({ }^{\circ} \mathrm{C}\right)$ & 14,62 & 14,45 & 16,33 & 16,07 & $\mathrm{C}=0,64+0,972 \mathrm{D} \mathrm{R}^{2}=0,94$ \\
\hline Umidade relativa no dossel $(\%)$ & 83,12 & 82,51 & $*$ & $*$ & $\mathrm{C}=13,2+0,848 \mathrm{D} \quad \mathrm{R}^{2}=0,89$ \\
\hline Umidade relativa nos cachos $(\%)$ & 83,84 & 84,87 & * & $*$ & $\mathrm{C}=23,1+0,719 \mathrm{D} \quad \mathrm{R}^{2}=0,74$ \\
\hline RFA sobre o dossel $\left(\mathrm{MJ} \cdot \mathrm{m}^{-2} \cdot \mathrm{dia}^{-1}\right)$ & 5,38 & 7,98 & 4,32 & 7,63 & $\mathrm{C}=0,629 \mathrm{D} \quad \mathrm{R}^{2}=0,91$ \\
\hline RFA ao nível dos cachos (MJ.m ${ }^{-2} \cdot$ dia $\left.^{-1}\right)$ & 1,25 & 2,87 & 1,26 & 1,65 & $\mathrm{C}=0,570 \mathrm{D} \mathrm{R}^{2}=0,80$ \\
\hline Velocidade do vento diária $\left(\mathrm{m} . \mathrm{s}^{-1}\right)$ & 0,093 & 0,904 & 0,079 & 0,817 & $\mathrm{C}=0,107 \mathrm{D} \mathrm{R}^{2}=0,49$ \\
\hline
\end{tabular}

*ausência de dados por problemas nos termopares de bulbo úmido.

**Equações de regressão referentes à análise das variáveis micrometeorológicas dos dois ciclos em conjunto, pois não houve diferença significativa no comparativo entre ciclos.

A radiação fotossinteticamente ativa (RFA) ao nível do dossel vegetativo teve redução linear de $38 \%$ (coeficiente angular de 0,$629 ; \mathrm{P}_{\beta=1}<0,0001$ ). O efeito variou de um ciclo para outro, tendo uma redução de $33 \%$ no primeiro ciclo e de $43 \%$ no segundo ciclo, o que pode estar relacionado à perda de transparência do plástico ao longo do tempo (Tabela 1). Destaca-se que somente pela restrição da radiação fotossinteticamente ativa imposta pela cobertura, de acordo com a média de cada ciclo (Tabela 1), haveria redução média de $1,08 \mathrm{~mm} \mathrm{dia}^{-1}$ e $0,66 \mathrm{~mm} \mathrm{dia}^{-1}$ da lâmina de água evaporada nos ciclos 2005/06 e 2006/07, respectivamente, de acordo com a equivalência proposta por ALLEN et al. (1998).

Outros trabalhos conduzidos no Brasil, com diferentes tipos de cobertura no cultivo da videira, também demonstraram atenuação da radiação RFA (LULU et al., 2005). De acordo com FERREIRA et al. (2004), a RFA pode ser reduzida em até $85 \%$. VENTURIN \& SANTOS (2004) também observaram reduções de até $50 \%$ de RFA, quando utilizaram cobertura plástica de mesma composição e espessura semelhante àquela empregada no presente estudo $(200 \mu \mathrm{m})$, em vinhedo de 'Niágara Rosada' (Vitis labrusca L.) localizado em Caxias do Sul-RS, na mesma macrorregião produtora de uvas onde foi efetuado o presente trabalho. Esses autores salientam que as maiores reduções de RFA foram alcançadas em plásticos com quatro anos de uso. $\mathrm{Na}$ Itália, RANA et al. (2004) também observaram diminuição de RFA no dossel vegetativo, sendo atenuada em $17 \%$ por clarite e em $32 \%$ por plástico impermeável e translúcido. No presente trabalho, a redução observada foi de $56,45 \%$ e $23,64 \%$ no nível dos cachos, nos ciclos 2005/06 e 2006/07, respectivamente $\left(0,570 ; \mathrm{P}_{\beta=1}<00001\right)$ (Tabela 1). Essa diferença também está associada aos diferentes níveis de desfolha executados nos dois ciclos, sobretudo no primeiro ciclo, em que a retirada de folhas das videiras descobertas foi superior, o que acarretou maiores valores de interceptação de RFA (Tabela 1).

$\mathrm{Na}$ avaliação específica do período de maturação, foi observado o mesmo padrão do ciclo todo, pois as temperaturas máximas no vinhedo coberto tiveram incremento em relação ao descoberto, aumentando também as temperaturas médias (Figura 1). A mesma tendência foi observada na interceptação de RFA pela cobertura plástica. Contudo, no segundo ciclo, a interceptação da radiação no nível dos cachos foi menor que no ciclo 2005/06 (Figura 1), devido a diferenças no manejo de desfolha.

Observou-se que o plástico utilizado interceptou um percentual de $56 \%$ de radiação na faixa do ultravioleta (UV) (Tabela 2). Esse comprimento de onda é responsável pela degradação das coberturas e, principalmente, tem efeito nocivo aos vegetais (TAIZ \& ZEIGER, 2004). Depois do UV, as radiações nas faixas do azul e do verde foram as mais interceptadas, $49 \% \mathrm{e}$ $48 \%$, respectivamente (Tabela 2 ). Corroborando esses resultados, KITTAS et al. (1999) observaram que coberturas plásticas têm grande influência na diminuição da radiação, na faixa do azul. Sabe-se que a diminuição nessa faixa de radiação afeta vários processos nas plantas coordenados pelo criptocromo, tais como crescimento de ramos, caules e folhas,

Ciência Rural, v.39, n.7, out, 2009. 


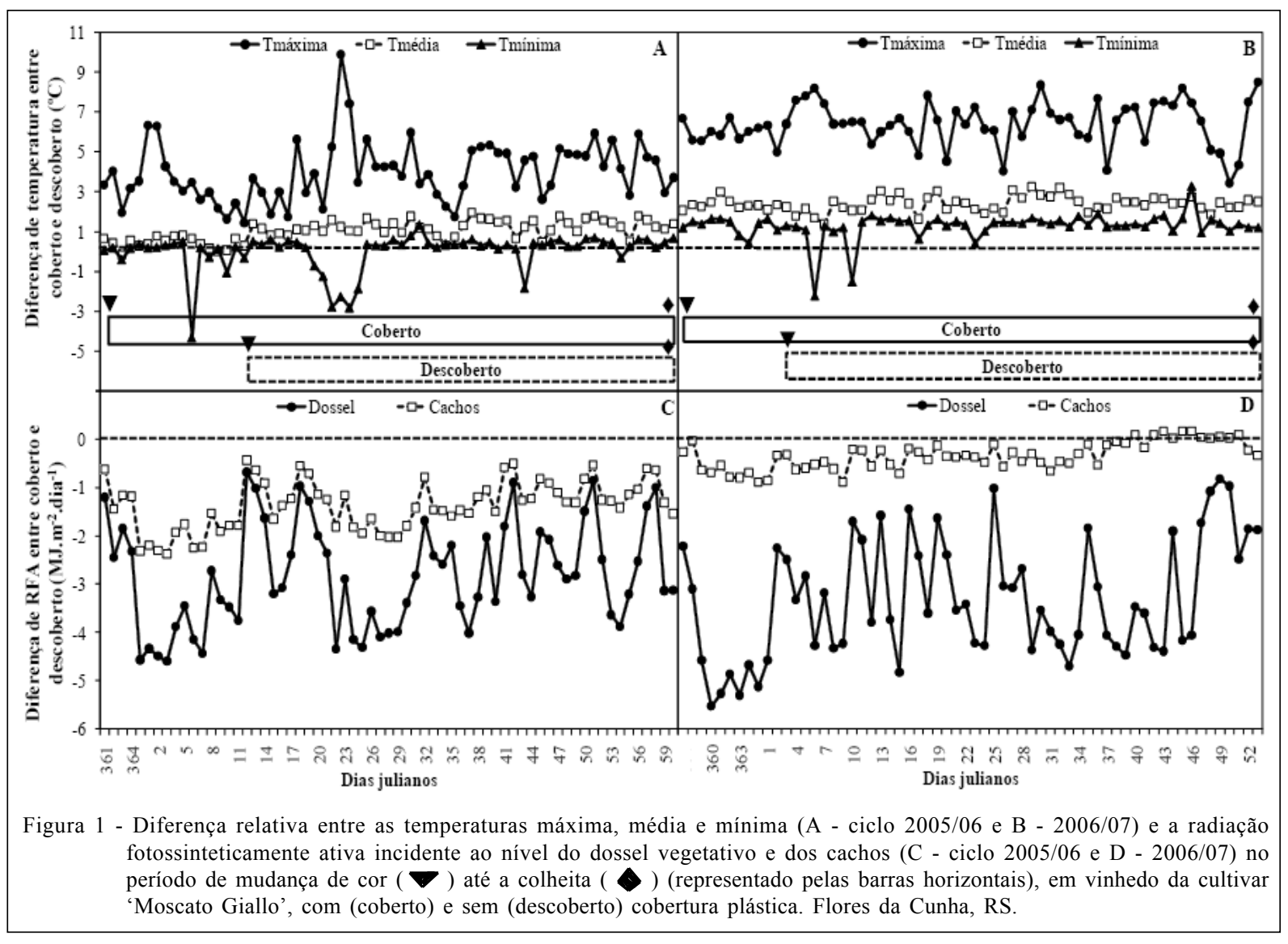

percepção do sinal luminoso e abertura estomática (TAIZ \& ZEIGER, 2004).

A razão vermelho:vermelho-distante foi reduzida pela cobertura plástica em 11,98\% (Tabela 2). Em condições de sombra, como nesse caso, a luz vermelha é mais atenuada que a faixa do vermelhodistante (ZANINE \& SANTOS, 2004), e a radiação difusa é rica em vermelho-distante (CASTRO et al., 2005). A diminuição da relação entre vermelho e vermelho-distante faz com que as plantas reconheçam condições de sombremento e se adaptem, por meio de alterações na anatomia foliar, incrementando o teor de pigmentos e a capacidade fotossintética (TAIZ \& ZEIGER, 2004).

\section{CONCLUSÕES}

O uso de coberturas plásticas impermeáveis sobre fileiras de plantas modifica o microclima, aumentando a temperatura do ar e diminuindo a radiação fotossinteticamente ativa e a velocidade do vento.

Esse tipo de cobertura plástica provoca também redução da radiação solar ultravioleta incidente e reduz a razão entre a radiação nas faixas do vermelho e do vermelho-distante.

Tabela 2 - Percentual de interceptação da radiação solar pela cobertura plástica sobre vinhedo da cultivar (Vitis vinifera L.) 'Moscato Giallo' e relação entre os comprimentos de onda vermelho e vermelho-distante (V:VD), medidos com espectroradiômetro. Flores da Cunha, RS.

\begin{tabular}{ccccccc}
\hline \multicolumn{5}{c}{ Comprimento de onda* } \\
\hline UV & Azul & Verde & Vermelho & Vermelho-distante & Radiação total & Relação V:VD \\
$56,18 \mathrm{a}^{* *}$ & $49,08 \mathrm{~b}$ & $44,85 \mathrm{~b}$ & $39,24 \mathrm{c}$ & $33,77 \mathrm{~d}$ & $45,09 \mathrm{~b}$ & 11,98 \\
\hline
\end{tabular}

*UV - ultravioleta (300-390nm), azul (450-490nm), verde (490-580nm), vermelho (620-700nm) e vermelho-distante (700-750nm).

**Médias seguidas de letras distintas diferem entre si em nível de significância de $5 \%$ de acordo com teste de Tukey. 


\section{REFERÊNCIAS}

ALLEN, R.G. et al. Crop evapotranspiration: guidelines for computing crop water requirement. Rome, Italy: FAO, 1998. 300p. (FAO Irrigation and Drainage Paper 56).

CARDOSO, L.S. et al. Alterações micrometeorológicas em vinhedos pelo uso de coberturas de plástico. Pesquisa Agropecuária Brasileira, v.43, n.4, p.441-447, 2008. Disponível em: <http://www.scielo.br/scielo.php?pid=S0100$204 X 2008000400001 \&$ script $=$ sci arttext\&tlng $=$ pt $>$. Acesso em: 28 nov. 2008 . doi: $10.1590 / \mathrm{S} 0100-204 \mathrm{X} 2008000400001$.

CARBONNEAU, A. Place du microclimat de la partie aérienne parmi les facteurs déterminant les productions viticoles. Bulletin de l'OIV, v.1, p.473-477, 1984.

CASTRO, E.M. et al. Aspectos anatômicos e fisiológicos de plantas de guaco submetidas a fotoperíodo. Horticultura Brasileira, v.23, n.3, p.846-850, 2005.

CHAVARRIA, G. et al. Incidência de doenças e necessidade de controle em cultivo protegido de videira. Revista Brasileira de Fruticultura, v.29, n.3, p.477-482, 2007. Disponível em: $<$ http://www.scielo.br/scielo.php? pid=S 0100 $29452007000300014 \&$ script $=$ sci_arttext\&tlng $=\mathrm{em}>$. Acesso em: 28 fev. 2009. doi: 10.1590/S0100-29452007000300014.

CRITTEN, D.L.; BAILEY, B.J. A review of greenhouse engeering developments during the 1990s. Agriculture and Forest Meteorology, v.112, p.1-22, 2002. Disponível em: $<\mathrm{ht} \mathrm{t} \mathrm{p} \mathrm{:} \mathrm{/} \mathrm{/} \mathrm{w} \mathrm{w} \mathrm{w.} \mathrm{s} \mathrm{c} \mathrm{i} \mathrm{e} \mathrm{n} \mathrm{c} \mathrm{e} \mathrm{d} \mathrm{i} \mathrm{r} \mathrm{e} \mathrm{t} \mathrm{.} \mathrm{c} \mathrm{o} \mathrm{m/}$ science?_ob=ArticleURL\&_udi=B6V8W-4619J3V$1 \&$ user $=3793194 \&$ rdoc $=1 \&$ fmt $=\&$ orig $=$ search \& so $\mathrm{rt}=\overline{\mathrm{d}} \& \mathrm{view}=\mathrm{c} \&$ a c ct $=\mathrm{C} 00006139 \overline{8} \&$ \& version $=\overline{1} \&$ _ u r l Versio $\bar{n}=0 \&$ u s e rid $=3793194 \&$ $\overline{\mathrm{md}} 5=\mathrm{f} 053 \mathrm{~b} 4 \mathrm{e} 3 \mathrm{acc} 481 \mathrm{f} 2 \mathrm{e} 8 \mathrm{~d} 7 \overline{6} \mathrm{~b} 4 \mathrm{cfade} 38 \mathrm{e} 0>$. Acesso em: 06 abr. 2009. doi: 10.1016/S0168-1923(02)00057-6.

FERREIRA, M.A. et al. Modificação parcial do ambiente de cultivo da videira 'Cabernet Sauvignon' sobre diferentes portaenxertos: efeito sobre a produção e o teor de sólidos solúveis. Bragantia, v.63, n.3, p.439-445, 2004. Disponível em: $<$ http:/ /www.scielo.br/scielo.php?script $=$ sci_arttext\&pid $=$ S000687052004000300014>. Acesso em: 28 fev. 2009. doi: 10.1590/ S0006-87052004000300014.

KITTAS, C. et al. Influence of covering material and shading on the spectral distribution of light in greenhouse. Journal of Agricultural Engineering Research, v.73, p.341-351, 1999.

KÖPPEN, W. Das geographische system der klimatologie. Berlim: Borntrager, 1936. 44p.

LULU, J. et al. Efeito do microclima na qualidade da uva de mesa 'Romana' (A 1105) cultivada sob cobertura plástica. Revista Brasileira de Fruticultura, v.27, n.3, p.422-425, 2005. Disponível em: <http://www.scielo.br/scielo.php?pid=S0100$29452005000300020 \&$ script $=$ sci_arttext\&tlng $=$ en $>$. Acesso em: 28 fev. 2009 . doi: $10.1590 / \bar{S} 0100-29452005000300020$.

PEDRO JÚNIOR, M.J. et al. Efeito do uso de quebra-ventos na produtividade da videira 'Niágara Rosada'. Revista Brasileira de Agrometeorologia, v.6, n.1, p.75-79, 1998.

PEZZOPANE, J.E.M. et al. Alterações microclimáticas causadas pelo uso de tela plástica, Engenharia Agrícola, v.24, n.1, p.9-
15, 2004. Disponível em: <http://www.scielo.br/ s cie 1 o.php? s c ript $=$ sci art text \& pid=S 0100 69162004000100002>. Acesso em: 07 abr. 2009. doi: 10.1590/ S0100-69162004000100002

R. DEVELOPMENT CORE TEAM. R: a language and environment for statistical computing. R. Foundation for statistical computing. Vienna, Austria. Disponível em: $<\mathrm{http}$ / /www.r-project.org/ >. On line. Acesso em: 01 jan. 2007.

RANA, G. et al. Microclimate and plant water relationship of the "overhead" table grape vineyard managed with three covering techniques. Scientia Horticulturae, v.102, p.105120, 2004. Disponível em: <http://www.sciencedirect.com/ science? ob=ArticleURL \& udi=B6TC3-4BVPTN4$3 \&$ user $=\overline{3} 793194 \&$ rdoc $=1 \&$ fmt $=\&$ orig $=$ search $\&$ sort $=\mathrm{d}$ $\& v \bar{i} w=\mathrm{c} \&$ acct $=\mathrm{C} \overline{0} 00061398 \&$ \& version $=1 \&$ \&urlVersion $=0 \&$ userid $=3793194 \& \mathrm{md} 5=0 \mathrm{fcc} 5 \mathrm{edc} 48 \mathrm{cbbf} 3 \mathrm{~d} 85 \mathrm{c} 61 \mathrm{~b} \overline{\mathrm{b}} 7048 \mathrm{~d} 30 \mathrm{eb}>$. Acesso em: 06 abr. 2009. doi: 10.1016/j.scienta.2003.12.008.

REIS, N.V.B.; CARRIJO, O.A. Estufa, material de cobertura e cortinamento - durabilidade e transparência à radiação solar. In: CONGRESSO BRASILEIRO DE AGROMETEOROLOGIA, 11., 1999, Florianópolis, SC. Anais... Florianópolis: Sociedade Brasileira de Agromteorologia, 1999. CD-ROM.

SEEMAN, J. Greenhouses climate. In: SEEMAN, J. et al. Agrometerology. New York: Springer-Verlag, 1979. p.167178 .

SEGOVIA, J.F.O. et al. Comparação do crescimento e desenvolvimento da alface (Lactuca sativa L.) no interior e no exterior de uma estufa de polietileno em Santa Maria, RS. Ciência Rural, v.27, n.1, p.37-41, 1997. Disponível em: $<$ http://www.scielo.br/scielo.php?script=sci_arttext\&pid=S0103$84781997000100007 \& \operatorname{lng}=$ pt\&nrm $=\mathrm{iso}>$. Acesso em: $28 \mathrm{fev}$. 2009. doi: 10.1590/S0103-84781997000100007.

SENTELHAS, P.C. et al. Efeitos de diferentes tipos de cobertura, mine estufas na atenuação da radiação solar e da luminosidade. In: CONGRESSO BRASILEIRO DE AGROMETEOROLOGIA, 10., 1997, Piracicaba. Anais... Piracicaba: Sociedade Brasileira de Agrometeorologia, 1997. p.480-484.

TAIZ, L.; ZEIGER, E. Fisiologia vegetal. 3 ed. Porto Alegre: Artmed, 2004. 719p.

TEIXEIRA, A.H. de C. et al. Relações entre o índice de área foliar e a radiação solar na cultura da videira. Revista Brasileira de Fruticultura, v.5, n.2, p.143-146, 1997.

STRECK, E.V. et al. Atualização da classificação taxonômica das unidades de mapeamento do levantamento de reconhecimento dos solos do estado do Rio Grande do Sul. Porto Alegre: EMATER, 1999. 5p.

TONIETTO, J.; FACALDE, I. Regiões vitivinícolas brasileiras. In: UVAS para processamento. Brasília: Embrapa Informação Tecnológica, 2003. 134p. (Frutas do Brasil, 34).

VENTURIN, M.; SANTOS, H.P. Caracterização microclimática e respostas fisiológicas de uvas de mesa (Vitis labrusca e Vitis vinifera) cultivadas em ambiente protegido. In: CONGRESSO BRASILEIRO DE FRUTICULTURA, 18., 2004. Florianópolis, SC. Anais... Florianópolis: Sociedade Brasileira de Fruticultura, 2004. CD-ROM

ZANINE, A. de M.; SANTOS, E.D. Competição entre espécies de plantas - uma revisão. Revista da Faculdade de Zootecnia, Veterinária e Agronomia. v.11, n.1, p.10-30, 2004.

Ciência Rural, v.39, n.7, out, 2009. 